\title{
A NOTE ON SOLITARY WAVES WITH VARIABLE SURFACE TENSION IN WATER OF INFINITE DEPTH
}

\author{
E. ÖZUĞURLU ${ }^{\Xi 1}$ and J.-M. VANDEN-BROECK ${ }^{2}$
}

(Received 12 February, 2004; revised 29 November, 2006)

\begin{abstract}
Two-dimensional gravity-capillary solitary waves propagating at the surface of a fluid of infinite depth are considered. The effects of gravity and of variable surface tension are included in the free-surface boundary condition. The numerical results extend the constant surface tension results of Vanden-Broeck and Dias to situations where the surface tension varies along the free surface.
\end{abstract}

2000 Mathematics subject classification: primary 74J35, 76B45, 76D45, 76B 15.

Keywords and phrases: solitary waves, free-surface potential flows, capillarity (surface tension), gravity waves.

\section{Introduction}

Over the last 20 years, much progress has been achieved in the understanding of the effect of surface tension on solitary waves. In particular two new classes of solitary waves have been discovered. The first class consists of waves which possess a train of ripples of constant amplitude in the far field. These waves are often referred to as generalised solitary waves to distinguish them from true solitary waves which are characterised by a flat profile in the far field. The second class consists of solitary waves with oscillatory decaying tails in the far field. There is also an extensive literature on periodic waves with surface tension (see for example [1], [4] and [7]). One main result is the existence of many different branches of periodic solutions with dimples on their free-surface profiles.

Both solitary and periodic waves are usually studied by assuming constant surface tension. Vanden-Broeck [8] showed that the theory of periodic capillary waves can

\footnotetext{
${ }^{1}$ Bahcesehir University, Faculty of Arts and Sciences, Department of Mathematics and Computer Sciences, 34100 Besiktas, Istanbul, Turkey; e-mail: ersin.ozugurlu@bahcesehir.edu.tr. ${ }^{2}$ School of Mathematics, University of East Anglia, Norwich NR4 7TJ, England, UK.

(C) Australian Mathematical Society 2006, Serial-fee code 1446-1811/06
} 
be generalised by allowing the surface tension to vary along the free surface. This work was extended by Özuğurlu and Vanden-Broeck [6] to include the effect of gravity. The analysis in [8] and [6] was restricted to periodic waves. In this paper we consider solitary waves and provide numerical evidence that there are solitary waves with decaying oscillatory tails in the presence of variable surface tension. When the surface tension is constant, these new solutions reduce to the solitary waves of the second type mentioned in the previous paragraph. We assume the fluid to be inviscid and incompressible and the flow to be irrotational. A frame of reference moving with the solitary wave is chosen and the flow is assumed to be steady in that frame.

An important question is how the surface tension varies along the free surface. For periodic waves, it can easily be shown that the surface tension has to vary periodically along the free surface (Vanden-Broeck [8]). This is not the case for solitary waves. For solitary waves, the curvature of the free-surface profile approaches zero in the far field. Therefore the effect of surface tension is negligible in the far field and we can assume without loss of generality a distribution of surface tension which approaches a constant value in the far field. We experimented with various such distributions of surface tension and found that the results were qualitatively similar. We present results for only one distribution of surface tension.

The formulation is presented in Section 2. The numerical procedure is described in Section 3. The results are presented in Section 4.

\section{Formulation of the problem}

The formulation and numerical procedure follow closely that of Vanden-Broeck and Dias [9]. At large depth the flow is characterised by a uniform stream to the right with a constant velocity $U$. We assume that the flow is steady. Cartesian coordinates are introduced with the $x$-axis parallel to the velocity $U$ at large depth and the $y$-axis directed vertically upwards. Gravity $g$ is acting in the negative $y$ direction. The origin of the coordinates is chosen on the free surface and the flow is assumed to be symmetric with respect to the $y$-axis. The velocity potential function $\phi$ and the stream function $\psi$ are used as independent variables. Without loss of generality we set $\psi=0$ on the free surface and $\phi=0$ at the point $x=0$ on the free surface.

We assume that the surface tension $T$ varies along the free surface and approaches a constant value $T_{0}$ as $|x| \rightarrow \infty$. We found that the numerical results are qualitatively independent of the particular distribution of surface tension chosen. Therefore we shall only present results for one distribution of surface tension, namely

$$
T(\phi)=T_{0}+\tilde{\epsilon} \kappa(\phi),
$$

where $T_{0}>0$ and $\tilde{\epsilon}>0$ are given constants and $\kappa$ is the curvature of the free surface counted positive when the centre of curvature lies inside the fluid. Relation (2.1) 
defines $T$ as a function of $\phi$. This can be viewed as an inverse formation in the sense that $T$ is found as a function of $x$ at the end of the computations after $x(\phi)$ has been calculated. Since $|x| \rightarrow \infty$ and $\kappa(\phi) \rightarrow 0$ as $|\phi| \rightarrow \infty, T_{0}$ is the constant value of the surface tension in the far field. We define dimensionless variables taking $T_{0} / \rho U^{2}$ as the unit length and $U$ as the unit velocity. If $u$ and $v$ denote respectively the horizontal and the vertical components of the velocity, we write

$$
\begin{aligned}
f & =\phi+i \psi \\
z & =x+i y \\
u-i v & =\left(\frac{d z}{d f}\right)^{-1}=\frac{1}{x_{\phi}+i y_{\phi}} .
\end{aligned}
$$

We seek $x_{\phi}+i y_{\phi}$ as an analytic function of $f$, in the domain $\psi \leq 0$.

On the free surface the Bernoulli equation yields in terms of the dimensionless variables

$$
\frac{1}{2} \frac{1}{x_{\phi}^{2}+y_{\phi}^{2}}+\alpha y+[1+\tilde{\epsilon} \kappa(\phi)] \kappa(\phi)=B
$$

where

$$
\alpha=\frac{g T_{0}}{\rho U^{4}} \quad \text { and } \quad \kappa(\phi)=\frac{y_{\phi} x_{\phi \phi}-x_{\phi} y_{\phi \phi}}{\left(x_{\phi}^{2}+y_{\phi}^{2}\right)^{3 / 2}}
$$

Here $B$ is the Bernoulli constant and $\rho$ is the density of the fluid.

We now apply Cauchy's integral formula to $x_{\phi}-1+i y_{\phi}$ on a path consisting of the free surface $\psi=0$ and a semicircle of arbitrarily large radius in the lower half $(\phi, \psi)$-plane. Since $x_{\phi}-1+i y_{\phi} \rightarrow 0$ as $\psi \rightarrow-\infty$, we have for $\psi<0$

$$
x_{\phi}-1+i y_{\phi}=-\frac{1}{2 \pi i} \int_{-\infty}^{+\infty} \frac{\left.\left(x_{\xi}-1+i y_{\xi}\right)\right|_{\psi=0}}{\xi-f} d \xi, \quad \psi<0 .
$$

Setting $\psi=0$ in (2.7) and taking the real part of the resulting expression then yields

$$
x_{\phi}=1-\frac{1}{\pi} \int_{-\infty}^{+\infty} \frac{y_{\xi}}{\xi-\phi} d \xi, \quad \psi=0,
$$

the integral being of Cauchy principal-value form. We use the symmetry of the flow to rewrite (2.8) as

$$
x_{\phi}=1-\frac{1}{\pi} \int_{0}^{\infty} y_{\xi}\left[\frac{1}{\xi-\phi}+\frac{1}{\xi+\phi}\right] d \xi .
$$

The integral in (2.9) is a Cauchy principal value. 
Relations (2.5) and (2.9) define a nonlinear integro-differential equation for $x_{\phi}+i y_{\phi}$ on the free surface. Upon solving this system, the free-surface profile is readily determined. The amplitude of the free-surface displacement is characterised by the distance $A$ between the origin of the coordinates and the level of the free surface at infinity. Thus

$$
A=-y \quad \text { at } \quad|\phi|=\infty, \quad \psi=0 .
$$

The definition (2.10) implies that $A>0$ for elevation free-surface profiles and $A<0$ for depression free-surface profiles.

For given values of $A$ and $\tilde{\epsilon}$ the system ((2.5), (2.9)) is discretised and the resulting algebraic equations are solved using Newton's method. The value of $\alpha$ is found as part of the solution. Details are given in Section 3.

We conclude by mentioning that the relation between the inviscid problem considered here and the viscous problem at high Reynolds number is described in LucassenReynders and Lucassen [5] and Özuğurlu and Vanden-Broeck [6].

\section{Numerical procedure}

In this section a numerical scheme is derived to solve the system ((2.5), (2.9)). The approach is similar to that used by Vanden-Broeck and Dias [9].

We define the $N$ mesh points $\phi_{I}=(I-1) E, I=1, \ldots, N$, and the $N-1$ midpoints $\phi_{I}^{M}=E / 2+(I-1) E, I=1, \ldots, N-1$. Here $E$ is the interval of discretisation. The unknowns are $y_{I}^{\prime}=y_{\phi}\left(\phi_{I}\right), I=1, \ldots, N$. Equation (2.9) is satisfied at the points $\phi_{1}^{M}$. The integral is evaluated by the trapezoidal rule with a sum over the points $\phi_{l}$. The symmetry of the quadrature and of the discretisation enables us to evaluate the Cauchy principal value as if it were an ordinary integral. This gives $x_{\phi}\left(\phi_{I}^{M}\right)$ in terms of the unknowns $y_{I}^{\prime}$. We evaluate $y_{\phi \phi}\left(\phi_{I}^{M}\right), x_{\phi \phi}\left(\phi_{I}^{M}\right)$ and $y_{\phi}\left(\phi_{I}^{M}\right)$ in terms of $y_{I}^{\prime}$ by finite differences and interpolation. Next the values of $y\left(\phi_{I}^{M}\right)$ in terms of $y_{1}^{\prime}$ are found by integrating $y_{l}^{\prime}$ by the trapezoidal rule. All these expressions are substituted in (2.5) which is evaluated at $\phi_{I}^{M}, I=1, \ldots, N-2$. This leads to $N-2$ equations for the $N$ unknowns $y_{I}^{\prime}, I=1, \ldots, N$. The last two equations are obtained by imposing the symmetry condition $y_{1}^{\prime}=0$ and the truncation condition $y_{N}^{\prime}=0$. For fixed values of $\tilde{\epsilon}$ and $\alpha$, this system of $N$ nonlinear algebraic equations with $N$ unknowns is solved by Newton's method.

\section{Discussion of results}

We used the numerical procedure described in Section 3 to compute solutions for various values of $\tilde{\epsilon}$ and $A$. In most of the computations presented we used $E=0.19$ 


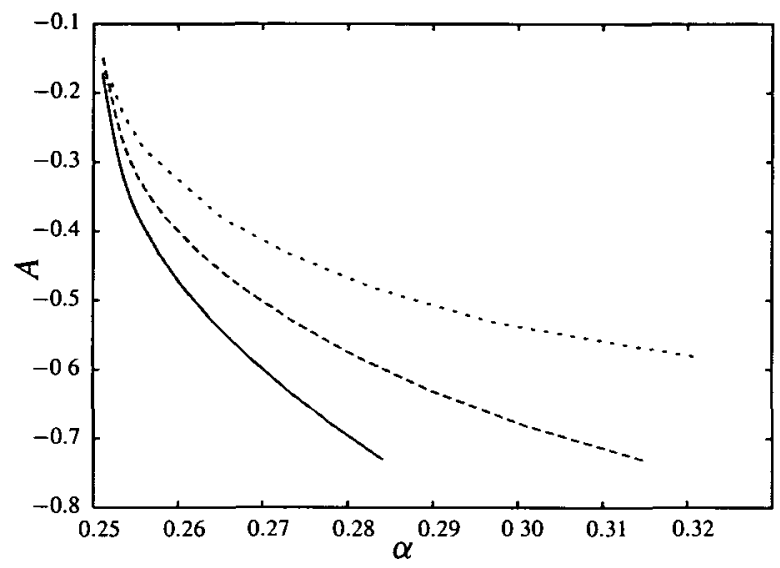

FIGURE 1. Values of $A$ versus $\alpha$. The curves from bottom to top correspond to $\tilde{\epsilon}=0, \tilde{\epsilon}=0.2$ and $\tilde{\epsilon}=0.5$.

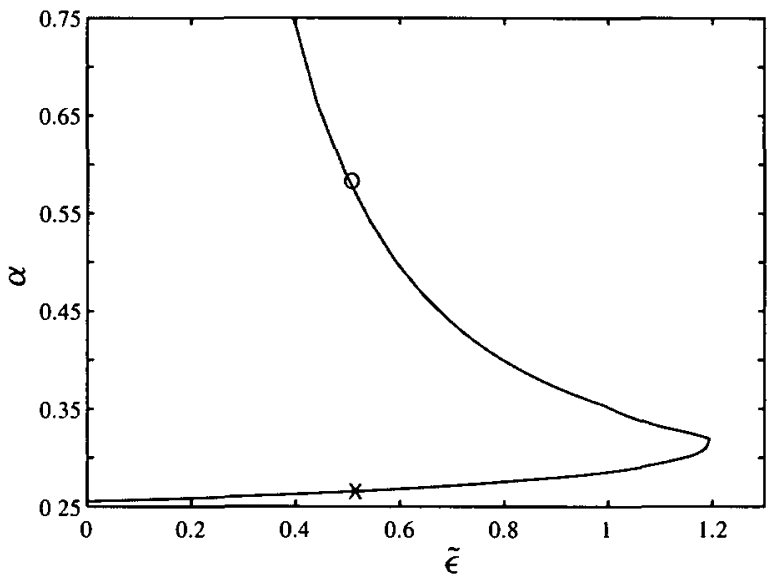

FIGURE 2. Values of $\alpha$ versus $\tilde{\epsilon}$ for $A=-0.381$.

and $N=400$. We repeated the calculations with different values of $E$ and $N$ and checked that the results are independent of $E$ and $N$ within graphical accuracy.

Some insight into the problem can be gained by using the classical linear theory of gravity capillary waves. Since $T \rightarrow T_{0}$ as $|x| \rightarrow \infty$, linear theory predicts that the free-surface elevation is of the form

$$
y=\operatorname{Re} C e^{i k x} \quad \text { as } \quad|x| \rightarrow \infty,
$$

where $C$ is a complex constant and $k$ is the wavenumber. The wavenumber $k$ satisfies the dispersion relation

$$
U^{2}=g / k+T_{0} k / \rho
$$


Using dimensionless variables, (4.2) is rewritten as

$$
k^{2}-k+\alpha=0
$$

Relation (4.3) is a quadratic equation for $k$ whose solutions are

$$
k_{ \pm}=\frac{1}{2}\left[1 \pm(1-4 \alpha)^{1 / 2}\right]
$$

For $\alpha<1 / 4$, the two solutions (4.4) are real and (4.3) predicts a train of periodic waves of constant amplitude. For $\alpha>1 / 4$, the roots (4.4) are complex conjugate and we can rewrite $(4.1)$ as

$$
y=e^{-\delta|x|} \operatorname{Re} C e^{i \beta x}
$$

where

$$
\beta=1 / 2, \quad \delta=(4 \alpha-1)^{1 / 2} / 2
$$

Relation (4.5) predicts a train of waves whose amplitudes tend to zero as $|x| \rightarrow \infty$.

Solitary waves with decaying oscillatory tails have to approach a uniform stream in the far field. Therefore their far field behaviour is a small perturbation of a uniform stream which can be described by the linear formula (4.5). The above results suggest that solitary waves with decaying oscillatory tails bifurcate from a uniform stream at the value $\alpha=1 / 4$ and exist for values $\alpha>1 / 4$. This was established numerically in the case of constant surface tension by Vanden-Broeck and Dias [9] and Dias, Menasce and Vanden-Broeck [3] and through rigorous analysis by several investigators (see Dias and Khariff [2] for further references and a review of the topic). As $\alpha \rightarrow 1 / 4, \delta \rightarrow 0$ and the solitary wave approaches a train of periodic waves of vanishing amplitude. Vanden-Broeck and Dias [9] and Dias, Menasce and Vanden-Broeck [3] computed both elevation and depression solitary waves. Stability analysis indicates that only the depression solitary waves are stable. Therefore we concentrate our attention on depression solitary waves.

The above discussion suggests that the branches of depression solitary waves with variable surface tension bifurcate from $\alpha=1 / 4$. This is confirmed by the numerical results of Figure 1 where we present values of $A$ versus $\alpha$ for various values of $\tilde{\epsilon}$. The curve for $\tilde{\epsilon}=0$ corresponds to the case of constant surface tension. The corresponding solutions were found to agree with those obtained by Vanden-Broeck and Dias [9]. This constitutes a check on the validity of our computer code. For a given value of $\alpha,|A|$ decreases as $\tilde{\epsilon}$ increases. We note that (2.1) implies that an increase in $\tilde{\epsilon}$ corresponds to an increase in the variable part of the surface tension $T$.

An interesting question is what ultimately happens when we increase $\tilde{\epsilon}$. It is found that for given values of $A$, there is a maximum value of $\tilde{\epsilon}$ such that there are no solutions for larger values of $\tilde{\epsilon}$. We will refer to this maximum value as $\tilde{\epsilon}_{\max }$. 


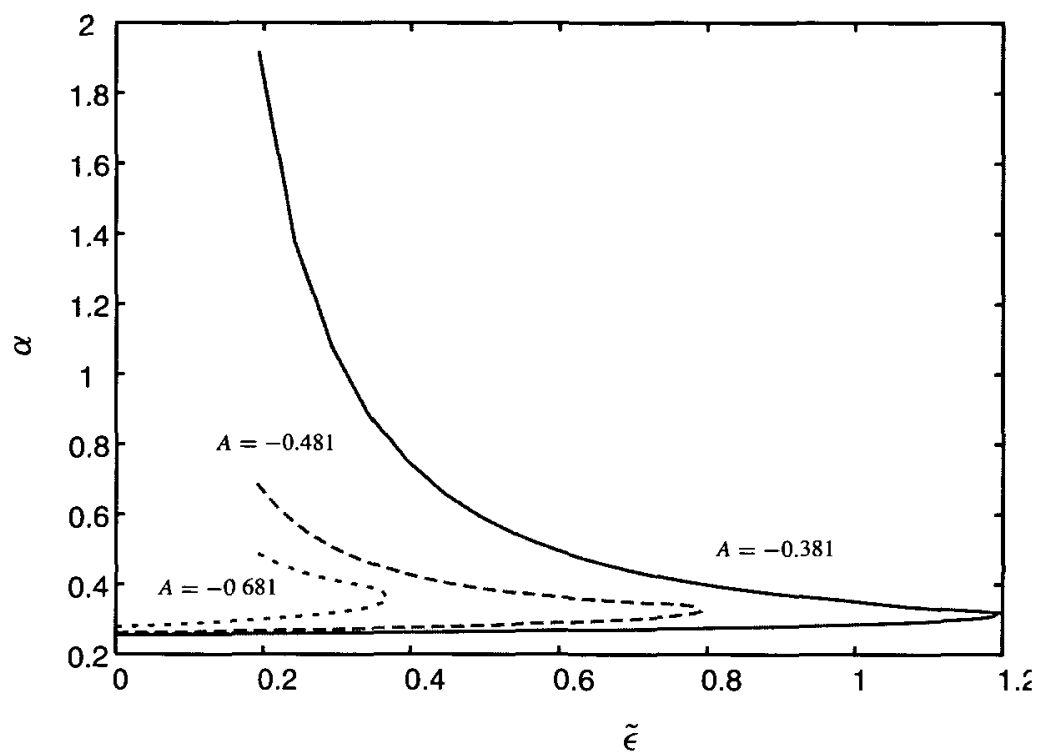

FIGURE 3. Values of $\alpha$ versus $\tilde{\epsilon}$ for $A=-0.381,-0.481$ and -0.681 . The curves move to the left as $A$ decreases.

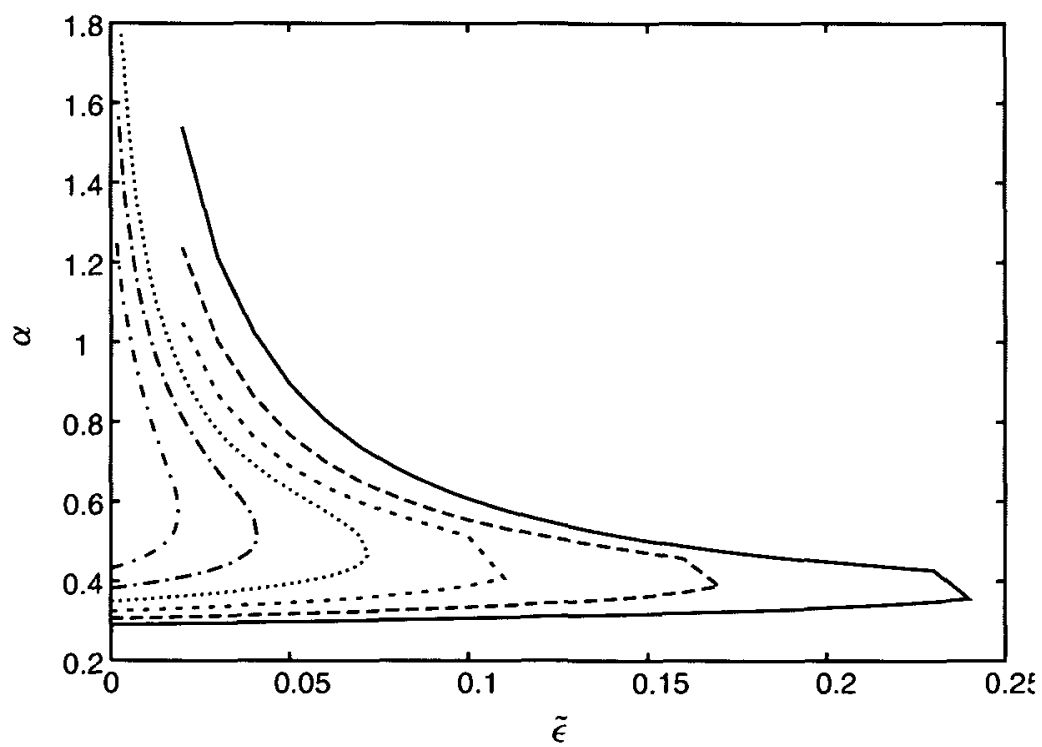

FIGURE 4. Values of $\alpha$ versus $\tilde{\epsilon}$ from $A=-1.281$ up to -0.781 by 0.1 increment. The curves move to the left as $A$ decreases. 


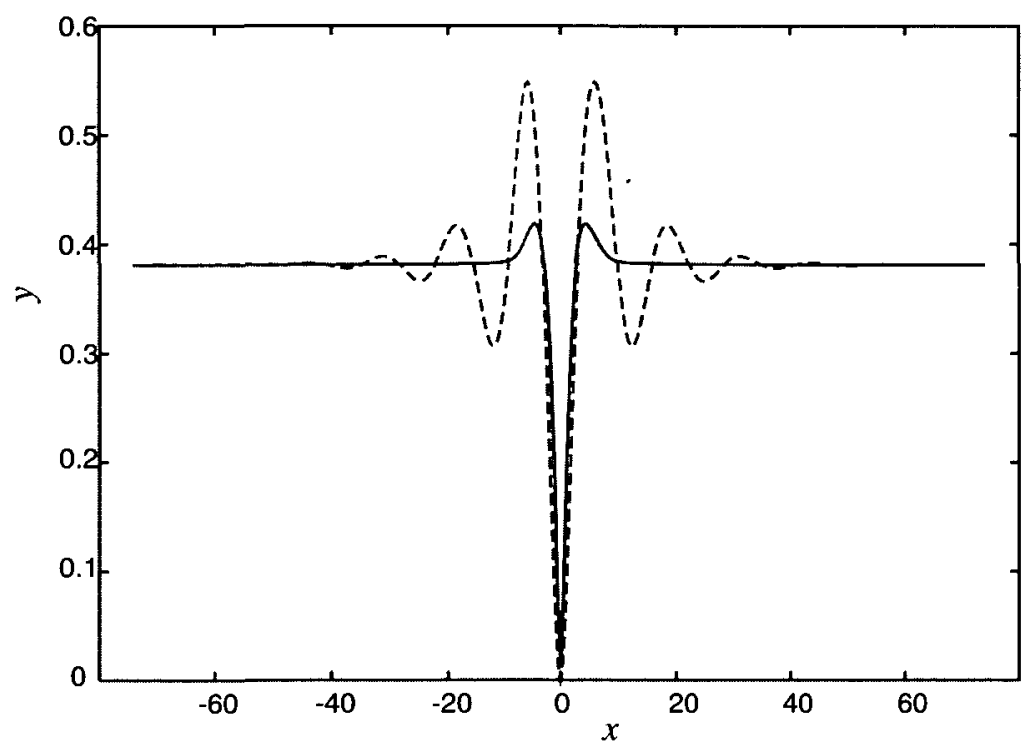

FIGURE 5. The two free-surface profiles for $A=-0.381$ and $\tilde{\epsilon}=0.5$. The broken line corresponds to the cross in Figure 2 and the solid line corresponds to the circle in Figure 2.

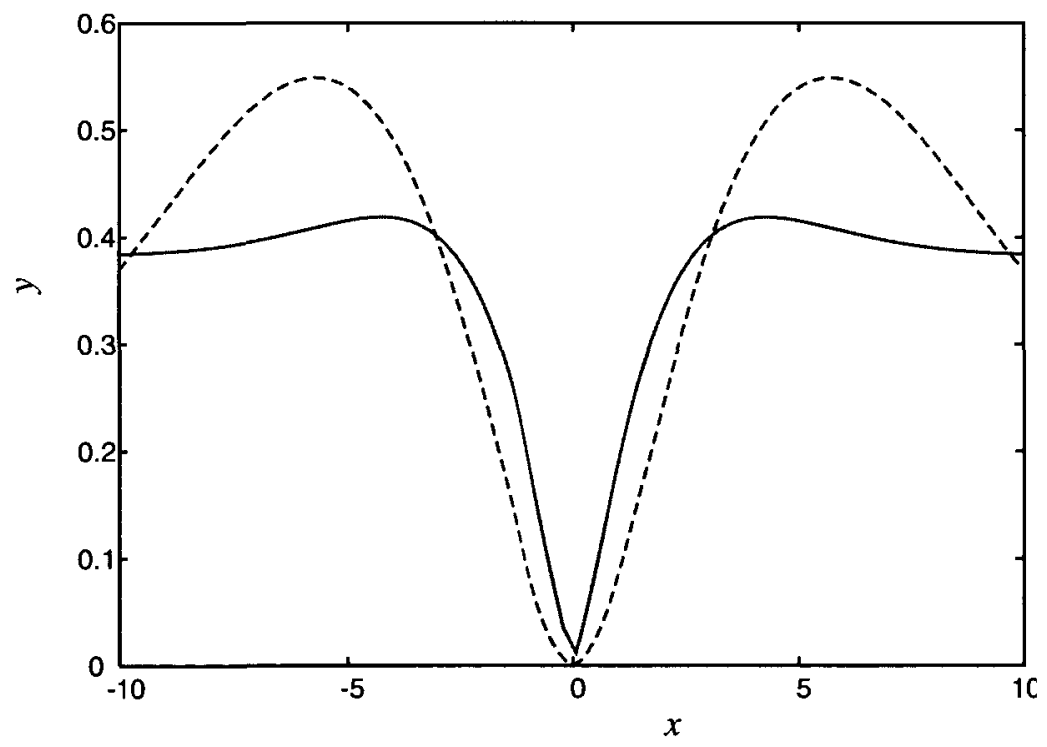

FIGURE 6. A close up of Figure 5 for $A=-0.381$ and $\tilde{\epsilon}=0.5$. The broken line corresponds to the cross in Figure 2 and the solid line corresponds to the circle in Figure 2. 


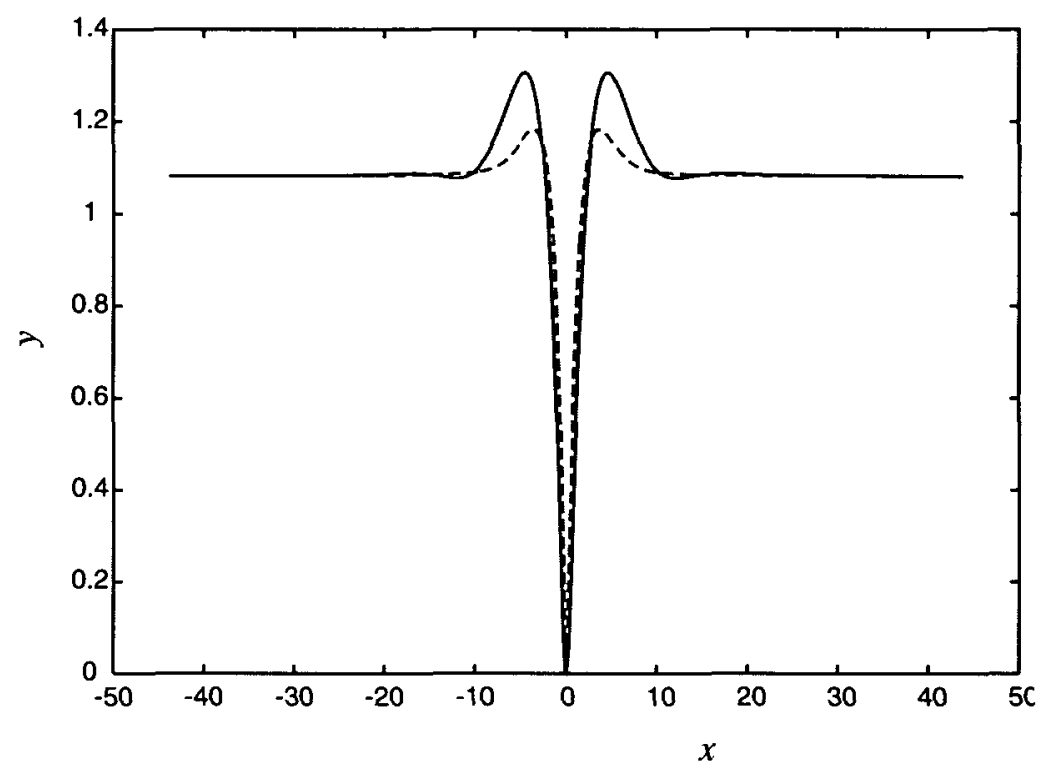

FIGURE 7. The two free-surface profiles for $A=-1.081$ and $\tilde{\epsilon}=0.036$.

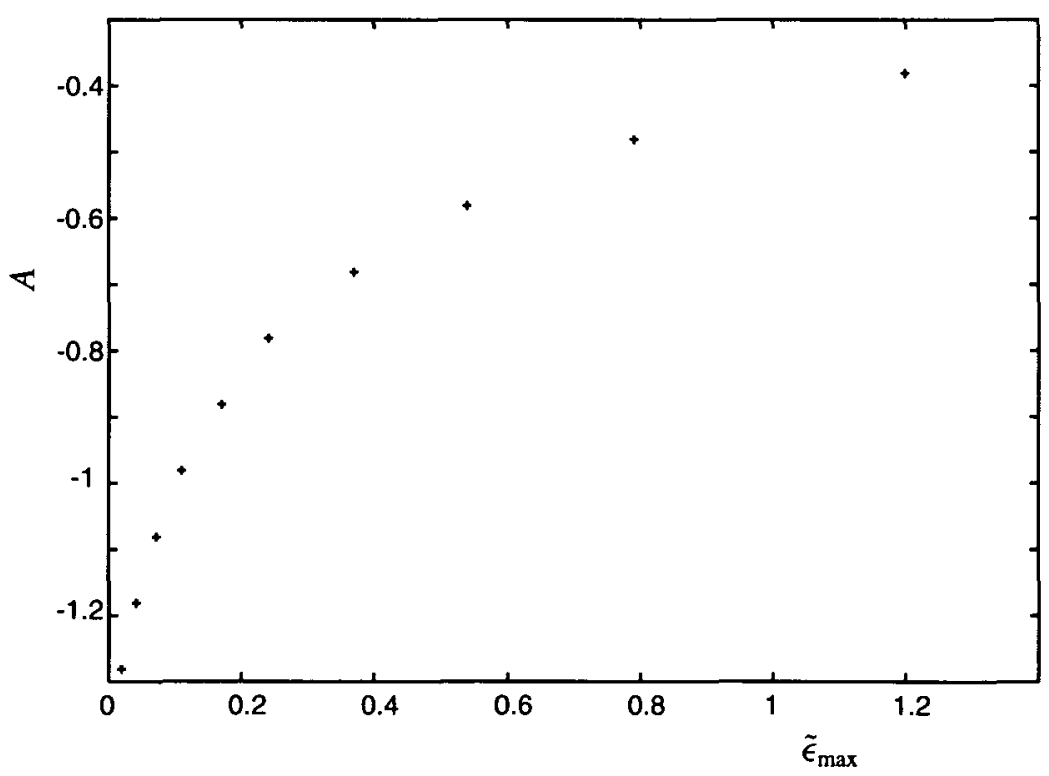

FIGURE 8. Values of $\tilde{\epsilon}_{\max }$ versus $A: \tilde{\epsilon}_{\max }$ decreases as $A$ decreases. 


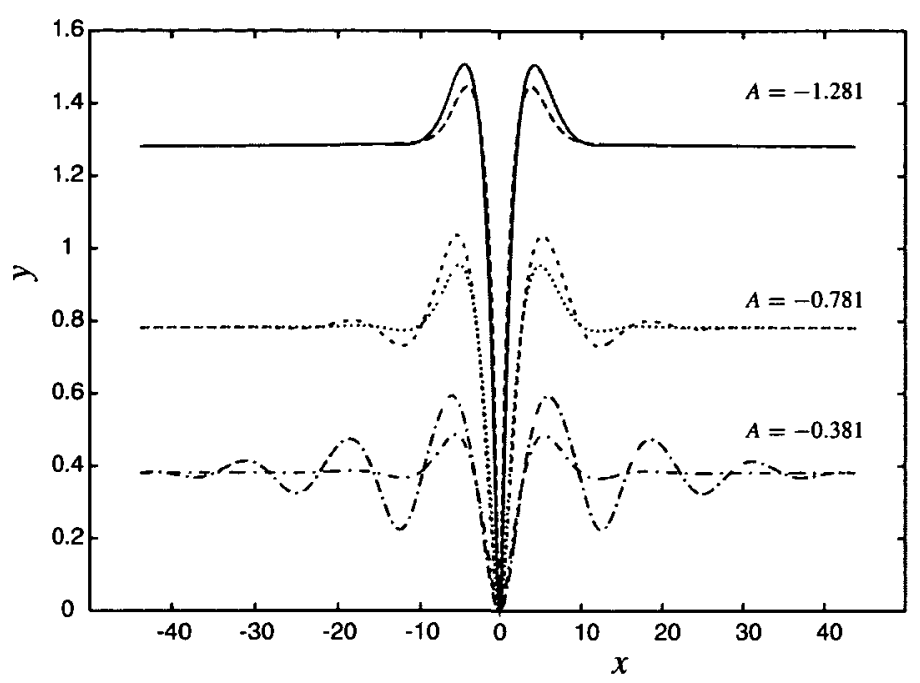

FIGURE 9. The free-surface profiles for $\tilde{\epsilon}=0$ and $\tilde{\epsilon}_{\max }$ when $A=-0.381,-0.781$ and -1.281 . For each $A$, the top curve corresponds to $\tilde{\epsilon}=0$ and the lower curve corresponds to $\tilde{\epsilon}_{\max }$. The crest of each curve goes down as $\tilde{\epsilon}$ increases.

This is shown in Figures 2-4. This finding implies that there are two possible solutions for some values of $\tilde{\epsilon}$. The profiles corresponding to two such solutions are shown in Figure 5 and expanded in Figure 6 for $\tilde{\epsilon}=0.5$ when $A=-0.381$. In Figure 7 , we show the corresponding solution for $A=-1.081$ and $\tilde{\epsilon}=0.036$.

Values of $\tilde{\epsilon}_{\max }$ are plotted as a function of $A$ in Figure 8 . We find that $\tilde{\epsilon}_{\max }$ decreases as $|A|$ increases. In other words, the upper bound $\tilde{\epsilon}_{\max }$ on the admissible values of $\tilde{\epsilon}$ (that is, on the magnitude of the variable part of $T$ in (2.1)) decreases as the amplitude $|A|$ increases.

We also plot the solution profiles for $\tilde{\epsilon}=0$ and $\tilde{\epsilon}_{\max }$ for some values of $A$ in Figure 9. The profiles show that the 'waviness' of the profiles increases as $|A|$ decreases. This can be explained by recalling that $\alpha \rightarrow 1 / 4$ as $|A| \rightarrow 0$. It then follows from (4.6) that the rate of decay $\delta$ decreases as $|A|$ decreases. We also note that for a given value of $|A|$ the waviness is less pronounced for $\tilde{\epsilon}_{\max }$ than for $\tilde{\epsilon}=0$. Thus variable surface tension tends to make the profiles flatter.

\section{Conclusions}

Previous results have demonstrated numerically and analytically the existence of solitary waves with decaying oscillatory tails. These studies assume that surface tension is constant. In this paper we have shown that there are solitary waves with decaying oscillatory tails in the case of variable surface tension. These results com- 
bined with those in [6] and [8] show that the assumption of constant surface tension is not crucial for the existence of gravity capillary periodic and solitary waves. All the branches of solutions bifurcate from a uniform stream at $\alpha=1 / 4$. It is found that for a given value of $|A|$ there is an upper bound on the magnitude $\tilde{\epsilon}$ of the variable part of the surface tension.

\section{References}

[1] B. Chen and P. G. Saffman, "Steady gravity-capillary waves on deep water. II. Numerical results for finite amplitude", Stud. Appl. Math. 62 (1980) 95-111.

[2] F. Dias and C. Khariff, "Nonlinear gravity and capillary-gravity waves", Ann. Rev. Fluid Mech. 31 (1999) 301-346.

[3] F. Dias, D. Menasce and J.-M. Vanden-Broeck, "Numerical study of capillary-gravity solitary waves", Eur. J. Mech. B Fluids 15 (1996) 17-36.

[4] S. J. Hogan, "Some effects of surface tension on steep water waves. Part 3", J. Fluid Mech. 110 (1981) 381-410.

[5] E. H. Lucassen-Reynders and J. Lucassen, "Properties of capillary waves", Adv. Colloid Interface Sci. 2 (1969) 347-395.

[6] E. Özuğurlu and J.-M. Vanden-Broeck, "The influence of variable surface tension on capillarygravity waves", J. Eng. Math. 40 (2001) 269-282.

[7] L. W. Schwartz and J.-M. Vanden-Broeck, "Numerical solution of the exact equations for capillarygravity waves", J. Fluid Mech. 95 (1979) 119-139.

[8] J.-M. Vanden-Broeck, "Capillary waves with variable surface tension", Z. Angew. Math. Phys. 47 (1996) 799-808.

[9] J.-M. Vanden-Broeck and F. Dias, "Gravity-capillary solitary waves in water of infinite depth and related free-surface flows", J. Fluid Mech. 240 (1992) 549-557. 